\title{
Subband Extraction Strategies in Ship Detection with the Subaperture Cross-correlation Magnitude
}

\author{
Camilla Brekke, Member, IEEE, Stian Normann Anfinsen, Member, IEEE, and Yngvar Larsen, Member, IEEE
}

\begin{abstract}
The subaperture cross-correlation magnitude (SCM) has previously been proposed as a statistic which improves the contrast between small ship targets and the surrounding sea in synthetic aperture radar (SAR) images. This preprocessing technique utilizes the fast decorrelation of open water surface ripples on the scale of the SAR wavelength relative to coherent targets such as a ship. However, optimisation of the bandwidth splitting in the subband extraction has not received any attention. The aim of this study is twofold: (i) to describe the technical details of the algorithm, including modifications that are necessary to allow overlapping subapertures, and (ii) to study the effect of splitting the bandwidth into two azimuth subapertures with respect to varying bandwidths and subaperture overlap. The impact on the SCM is investigated in terms of measures of speckle reduction and target-to-clutter contrast. Experiments are performed on real single-look complex (SLC) SAR data containing repeated acquisitions of a vessel in open sea. The results indicate that the subband extraction strategy has a clear impact on performance.
\end{abstract}

Index Terms-synthetic aperture radar, target detection, subaperture processing, contrast enhancement.

\section{INTRODUCTION}

The cross-correlation of subaperture measurements from SAR was first suggested by Arnaud [1] to obtain an image product which alleviates the detection of difficult man-made targets with low backscattering coefficients and low contrast to the background sea clutter. The concept was later elaborated by Souyris et al. [2], who referred to the format as the twolook internal Hermitian product (2L-IHP). They extended the technique to polarimetric data and provided details on the implementation.

A subaperture is defined as a subset of the available aperture, which can be extracted as a subband in the frequency domain and focused into an image of complex scattering coefficients with degraded resolution. The subapertures are filtered out from the spectrum of full resolution SLC data. Since they correspond to separate parts of the range or azimuth frequency band, the subapertures will originate from different time slots. The idea is to compute a cross-correlation between the complex scattering coefficients of two subapertures, and use the magnitude as a test statistic for target detection.

Manuscript received on 2 May 2012 and revised on 22 August 2012

Camilla Brekke and Stian Normann Anfinsen are with the Department of Physics and Technology, University of Troms $\varnothing$, Norway (e-mail: Camilla.Brekke@uit.no). Yngvar Larsen is with the Northern Research Institute, Tromsø, Norway.

Colour versions of one or more of the figures in this paper are available online at http://ieeexplore.ieee.org.

Digital Object Identifier xx.xxxx/LGRS.xxxx.Xxxxxxx
The underlying principle is that open water decorrelates in less than $0.05 \mathrm{~s}$ at C-band ( $<0.1 \mathrm{~s}$ at L-band) [3], while a man-made target like a ship should remain coherent over the time separating the acquisition of the subapertures. Therefore, the SCM is expected to provide a higher contrast between ships and open water than ordinary single-look intensity images. This has effectively been demonstrated [2], [4]. Other approaches have been suggested, that correlate subaperture amplitude or intensity and discard the phase information [3], [5], [6], but they are not discussed here.

The original approach of Souyris et al. [2] produced two SCM images: one based on subapertures extracted in range and a second with subapertures extracted in azimuth direction. The magnitudes were subsequently added. However, it was found in a verification study [4] that azimuth band splitting is preferable, because it better preserves spatial resolution and target details. In this paper, we study an SCM algorithm limited to subband extraction from the azimuth spectrum only.

It was suggested in [1] that the SCM technique can be adapted to different sea conditions by adjusting the width of the extracted subbands, and also their spectral separation. A point target may present nonstationary behaviour throughout the illumination time, e.g., in cases of rough sea state [2]. This can make the target visible only within parts of the azimuth or range spectrum. According to [1], the width of the subbands might have to be reduced in agitated sea states to avoid decorrelation of the ship. It was further argued that both separation and subband width might have to be increased when the sea is calm, to enhance the decorrelation of the sea.

In spite of the incitements, the subband extraction strategy has so far not been studied in the literature on SCM applied to ship detection. Hence, this is the focus of this paper. We study the effect of splitting the azimuth spectrum into subapertures as a function of spectral bandwidth and overlap. We open for subaperture overlap to investigate the whole continuum of extraction strategies, ranging from narrow time-separated subapertures to full subaperture overlap. In addition, by utilizing more of the bandwidth, the loss of spatial resolution associated with subaperture processing can also be reduced.

Section II describes the details of the modified SCM algorithm which allows overlap of subaperture bandwidths, the subband extraction strategies, and the performance measures used in the experimental part. Section III describes the data set. Section IV presents and discusses the experimental results. The conclusions are given in section $\mathrm{V}$. 


\section{Methodology}

Souyris et al. [2] computed the SCM according to

$$
|\rho|=\left|\left\langle S_{1} \cdot S_{2}^{*}\right\rangle\right|
$$

where $*$ denotes the complex conjugate, $S_{1}$ and $S_{2}$ are the complex scattering coefficients associated with the two subapertures extracted for a given SLC pixel, $\langle\cdot\rangle$ denotes a spatial average in the vicinity of this pixel, and $|\cdot|$ is the determinant operator. The averaging was performed within a $3 \times 3$ neighborhood. In [2], two SCM images were produced by extracting subbands both in azimuth and range, and producing $\rho_{a z}$ and $\rho_{r g}$. The sum of the respective magnitudes, $\left|\rho_{a z}\right|+\left|\rho_{r g}\right|$, was then used as input to the target detection.

We confine the algorithm to splitting of the azimuth spectrum. Ship detection could subsequently be done by thresholding the azimuth SCM: $\left|\rho_{a z}\right|$. However, in case of overlap between the subapertures, $\left|\rho_{a z}\right|$ will be undersampled, causing aliasing and high-frequent noise that could degrade the performance of the algorithm. This important issue has not been properly treated in earlier studies of subaperture processing applied to ship detection. We propose to mitigate this by introducing an antialiasing filter in the processing chain. The algorithm to compute the improved product, denoted $\mathrm{SCM}^{+}$, is outlined next.

\section{A. Improved SCM ${ }^{+}$Algorithm}

The steps of the algorithm outlined in Fig. 1 are:

1) Data: The SLC SAR data used as input to the algorithm are converted to the frequency domain by a twodimensional fast Fourier transform (2D-FFT).

2) Spectrum shifted to Doppler centroid: It is known that the Earth's rotations causes systematic variations of the Doppler centroid along the satellites orbit [7]. We shift the Doppler centroid, $f_{d c}$, to zero azimuth frequency, $f_{0}$, to compensate for this effect. This is crucial for accurate subsequent processing.

3) Azimuth bandwidth reduction: A reduction of the azimuth bandwidht to $\sim 80 \%$ of the original is done to take into account that a modified Kaiser-Bessel weighting function has been applied in the processing of the delivered Radarsat-2 SLC products [8]. The reduced bandwidth is from here on referred to as the total available azimuth bandwidth, $B$, stretching from $f_{0}-B / 2$ to $f_{0}+B / 2$.

4) Subband extraction: Two azimuth subbands are extracted from $B$, that will be used to produce the subaperture scattering coeffifients $S_{1}$ and $S_{2}$ in step 6). The subband extraction strategies investigated in this study are described in section II-B.

5) Upsampling: To allow overlapping subapertures without introducing aliasing, both subapertures are first zeropadded to double size in both range and azimuth, then a low-pass filter is applied in step 7). Upsampling is done to increase the sampling frequency and avoid that unwanted components of the signal fall into the passband of the filter applied in 7). The centre frequencies of the subapertures, $f_{1}$ and $f_{2}$, are shifted to $f_{0}$ to avoid a frequency difference between them.

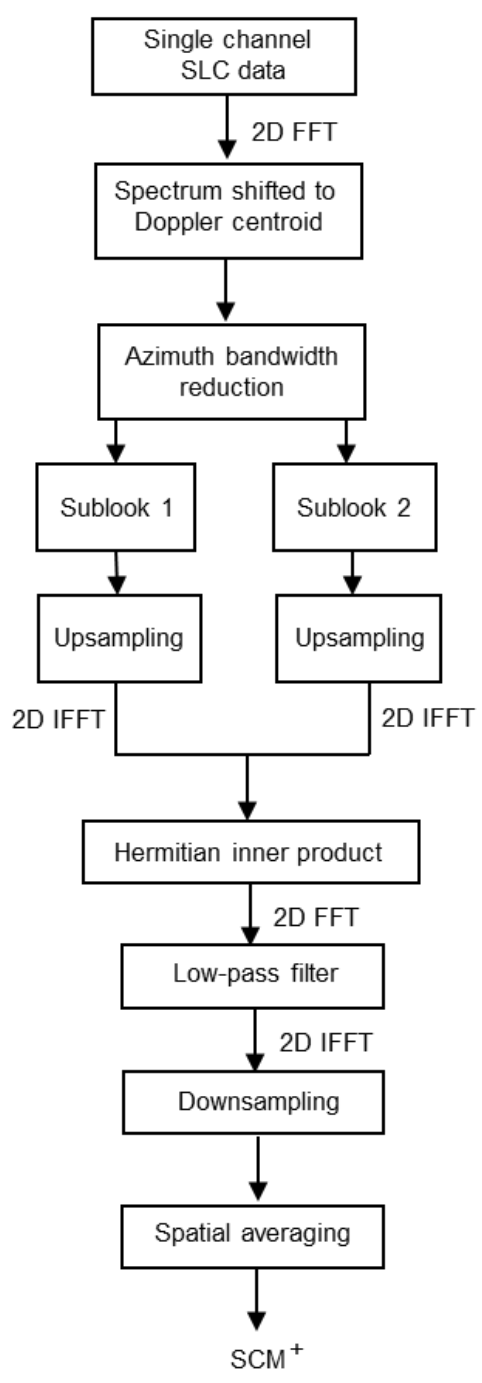

Fig. 1. Flow chart of the subaperture processing algorithm.

6) Hermitian inner product: A two-dimensional inverse fast Fourier transform (2D-IFFT) is used to produce $S_{1}$ and $S_{2}$. Their complex correlation is computed as the Hermitian inner product: $S_{1} \cdot S_{2}^{*}$.

7) Low-pass filter: After a 2D-FFT, a low-pass filter (e.g., a Hanning window) stretching from $f_{0}-B / 2$ to $f_{0}+B / 2$ is applied in both range and azimuth direction. Empiry shows better results when the low-pass filter is applied to the complex $\rho$, rather than the magnitude, $|\rho|$.

8) Downsampling: We return to the spatial domain by a 2DIFFT and downsample to half the size in both range and azimuth direction.

9) $S C M^{+}$: A local averaging filter (e.g., of size $3 \times 3$ pixels) is applied to the complex subaperture correlation, $\rho$. The magnitude of the data is extracted.

\section{B. Variation of Subaperture Bandwidth}

In the subband extraction stage, it is assumed that both subapertures are assigned the same fraction of the total available azimuth bandwidth $B$. The bandwidth allocated to each 


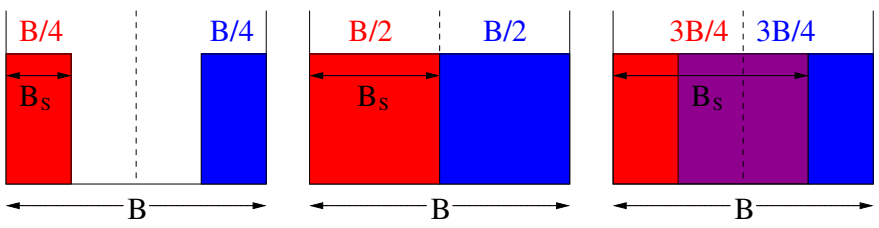

Fig. 2. Variation of the subaperture bandwidth $B_{S}$ within an azimuth spectrum with bandwidth $B$. The case $B_{S}=3 B / 4$ (right) has partial overlap between the subbands.

subaperture, denoted $B_{S}$, always starts from one end point of the azimuth spectrum and stretches towards the other, with a potential overlap with the other subaperture.

In the first limiting case, both subapertures have zero bandwidth. At a bandwidth of $B_{S}=B / 2$, the subapertures together occupy the whole azimuth spectrum, but there is no overlap. A subaperture bandwidth of $B_{S}=B$ defines the other limiting case, where there is a total overlap between the subapertures. It follows that $B_{S} \leq B / 2$ is equivalent to no overlap, $B / 2<B_{S}<B$ represents partial overlap, and the degree of overlap in the latter region is $2 B_{S} / B-1$. The bandwidth definition is illustrated by Fig. 2, which shows the cases: $B_{S}=B / 4, B_{S}=B / 2$, and $B_{S}=3 B / 4$.

The single-look intensity (SLI), which is commonly used in ship detection, is conventionally calculated as $I=S_{1} \cdot S_{1}^{*}$. This is equivalent to $\rho=S_{1} \cdot S_{2}^{*}$ with $B_{S}=B$ (thus $S_{1}=S_{2}$ ) and no averaging. Hence, unless countermeasures are taken, the quality of the SLI data will also be reduced by aliasing, as for the SCM product. To handle this, we apply the algorithm outlined in Fig. 1 for the limiting case $B_{S}=B$, but omit the local averaging in step 9). The improved SLI product, referred to as $\mathrm{SLI}^{+}$, is used as a reference case in section IV.

\section{Performance Measures}

In section IV, we compare $\mathrm{SCM}^{+}$to $\mathrm{SLI}^{+}$with respect to measures of the speckle level and the contrast between the target and the surrounding background. Specifically, we investigate the coefficient of variation (CV) and the targetto-clutter ratio (TCR). These parameters are measured as a function of the normalized subaperture bandwidth, defined as $\beta=B_{S} / B$. Hence, it ranges from 0 to 1 , while the region where the subapertures overlap goes from 0.5 to 1 .

1) Speckle Measure: The CV is a traditional measure of heterogeneity. It is the ratio of the standard deviation, $\sigma_{c}$, to the mean value, $\mu_{c}$, in a selected region of background clutter:

$$
C V=\frac{\sigma_{c}}{\mu_{c}} .
$$

An area of size $128 \times 128$ pixels is selected for all clutter regions studied.

2) Contrast Measure: The TCR is defined as the ratio of the mean value of the target region, $\mu_{t}$, to the mean value of the background clutter region, $\mu_{c}$ :

$$
T C R=\frac{\mu_{t}}{\mu_{c}} .
$$

The target region is delineated by manual segmentation. A background clutter region of size $128 \times 128$ pixels is selected in conjunction with every target studied.

\section{DATA SET}

The data set consists of three SAR SLC images and contains three acquisions of a search and rescue vessel (length: 64.5 $\mathrm{m}$, width: $13.8 \mathrm{~m}$ ) in open sea, see Table I. The SAR data were recorded over the Norne oil field in the Norwegian Sea. Experimental results based on this data set are discussed next.

TABLE I

QUAD-POLARIMETRIC RADARSAT-2 SAR IMAGERY AND RELATED AUXILIARY INFORMATION.

\begin{tabular}{l|l|l|l|l}
\hline Date & $\begin{array}{l}\text { Mode on asc- } \\
\text { ending orbit }\end{array}$ & $\begin{array}{l}\text { Wind speed } \\
\text { and direction }\end{array}$ & $\begin{array}{l}\text { Wave } \\
\text { height }\end{array}$ & $\begin{array}{l}\text { Inc. } \\
\text { angle }\end{array}$ \\
\hline 30 April 2010 & Fine Q26 & $7 \mathrm{~m} / \mathrm{s}, 20^{\circ} \mathrm{N}-\mathrm{NE}$ & $2.5 \mathrm{~m}$ & $45.3^{\circ}$ \\
4 August 2010 & Standard Q26 & $5 \mathrm{~m} / \mathrm{s}, 40^{\circ} \mathrm{NE}$ & $0.5 \mathrm{~m}$ & $45.4^{\circ}$ \\
5 August 2010 & Standard Q7 & $7 \mathrm{~m} / \mathrm{s}, 50^{\circ} \mathrm{NE}$ & $1.5 \mathrm{~m}$ & $27.1^{\circ}$ \\
\hline
\end{tabular}

\section{EXPERIMENTAL RESULTS}

Experiments show that the investigated target is often not properly resolved for $\beta \lesssim 0.15$. Hence, we limit the following analysis to $\beta \in[0.15,1]$.

\section{A. Speckle Statistics}

An analysis of the CV is used to investigate the impact of the subband extraction, and also of the antialiasing filter introduced in section II, on the speckle statistics in the $\mathrm{SLI}, \mathrm{SLI}^{+}, \mathrm{SCM}$ and $\mathrm{SCM}^{+}$images. The three polarimetric channels $\mathrm{HH}, \mathrm{HV}$, and VV are analyzed separately. The $\mathrm{VH}$ channel essentially contains the same information as $\mathrm{HV}$, and is therefore omitted.

Fig. 3 shows $\mathrm{CV}$ versus $\beta$ for open water measurements from the 30 April 2010 scene. The other scenes yield similar results. The highest pixel resolution which can be obtained is inversely proportional to the subaperture bandwidth. This holds for subband extraction in both range and azimuth direction [9]. We sample the image products at the limiting case resolution. Thus, as $\beta$ is raised, the $\mathrm{CV}$ is calculated from image data with steadily increasing resolution.

Fig. 3a shows the CV calculated from SCM and SLI images with no low-pass filter applied. Recall that the SLI product is equivalent to the $\mathrm{SCM}$ with $\beta=1$, but without spatial averaging. The dashed curves show that the CV for the SLI case is close to one in all channels, which is the theoretical value for exponentially distributed data [10, p. 88]. It is first observed that the CV values of the SCM data (solid lines) are on the whole significantly lower than one. This reflects that the spatial averaging in (1) obviously suppresses speckle, which is the main intention of the SCM approach. A second observation is that the curves exhibit a variation with $\beta$ containing certain pronounced features, which is discussed shortly.

Fig. $3 \mathrm{~b}$ shows the $\mathrm{CV}$ for all channels of the $\mathrm{SCM}^{+}$and $\mathrm{SLI}^{+}$images. The impact of the antialiasing filter is evident as the $\mathrm{SCM}^{+}$reaches lower $\mathrm{CV}$ values than the SCM (cf. Fig. 3a) as $\beta \rightarrow 1$, and the $\mathrm{CV}$ is lower for $\mathrm{SLI}^{+}$than for SLI. The difference between the $\mathrm{SLI}^{+}$and the $\mathrm{SCM}^{+}$again documents the effect of spatial averaging, although the reduction is lower 


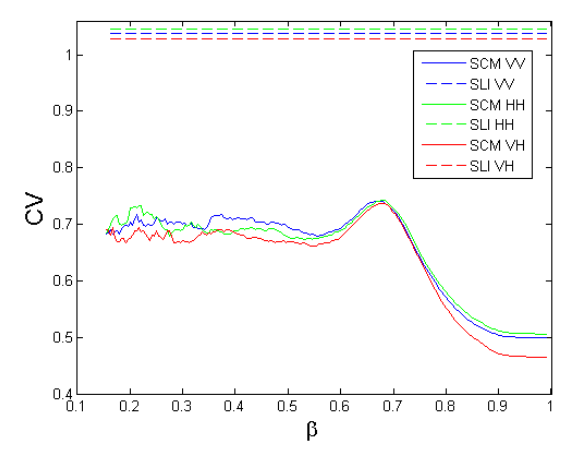

(a)

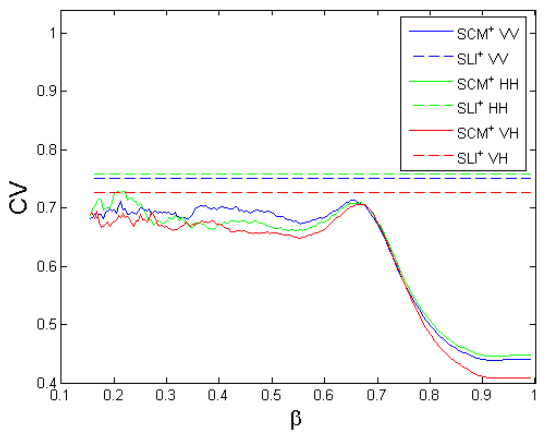

(b)

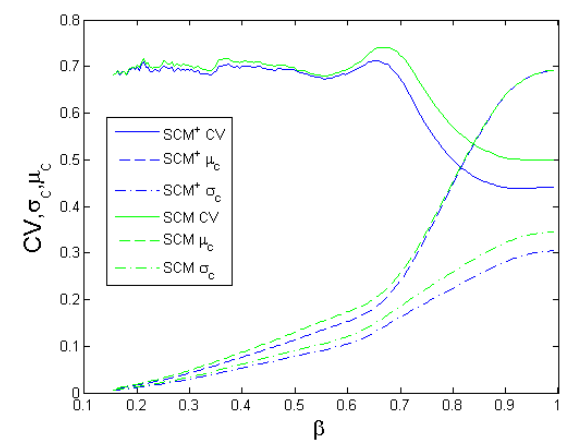

(c)

Fig. 3. $\mathrm{CV}$ versus normalized subaperture bandwidth $\beta$ : (a) SCM compared to SLI; (b) Improved $\mathrm{SCM}^{+}$compared to $\mathrm{SLI}^{+}$; (c) $\mathrm{The}_{\mathrm{VV}}$ channel $\mathrm{CV}$ of the $\mathrm{SCM}$ and $\mathrm{SCM}^{+}$products decomposed into $\mu_{c}$ and $\sigma_{c}$.

than in Fig. 3a. Further, the $\mathrm{CV}$ of the $\mathrm{SCM}^{+}$has the same variation with $\beta$ as for the SCM. We shall now explain the latter as an effect of the subaperture separation and overlap.

The subaperture separation decreases with $\beta$ and so the correlation between the subapertures increases, since the shorter time between the subaperture acquisitions implies more similar physical signal sources, and therefore higher coherence in both phase and amplitude. When $\beta>0.5$, there is even partial duplication of spectral signal content. We expect both the clutter mean, $\mu_{c}$, and standard deviation, $\sigma_{c}$, to increase with $\beta$. This is intuitive since $\mu_{c}$ follows the degree of correlation or coherence, and $\sigma_{c}$ follows $\mu_{c}$ due to the multiplicative signal model for radar speckle. This behaviour is exactly what is observed in Fig. 3c.

Fig. 3c displays $\mu_{c}$ and $\sigma_{c}$ alongside the CV for the SCM and $\mathrm{SCM}^{+}$products in the VV channel. Note that the $\mu_{c}$ and $\sigma_{c}$ have been scaled differently from the $\mathrm{CV}$, i.e., the former two have been multiplied by $10^{3}$. This is done to depict the variation of all entities in the same plot and to allow a study of their interrelation. The $\mu_{c}$ and $\sigma_{c}$ contribute as divisor and dividend to the $\mathrm{CV}$, according to Eq. (2), and the explicit decomposition is useful to interpret the variation of the CV curves. Specifically, the peak which appears within the subaperture overlap region can be explained when considering the relative slopes of $\mu_{c}$ and $\sigma_{c}$. Similar features are observed in the $\mathrm{CV}$ curves of other scenes, but the strength and the location on the $\beta$ axis vary.

Contrary to the subaperture separation, the subaperture bandwidth $(\beta)$ should not have any influence on the $\mathrm{CV}$, assuming the number of scatterers within the resolution cell is large. An increase in subaperture bandwidth means that additional information is made available, which is converted into better spatial resolution without affecting the speckle level. However, if the resolution was kept constant, then the extra bandwidth could be harvested in terms of improved speckle statistics. If stationary target properties are assumed over the entire resolution cell, then the mean value would stay the same, whereas the standard deviation would decrease.

Ouchi et al. have modelled the correlation of speckle intensity patterns in partly overlapping subapertures [3], [5]. This model assumed Rayleigh scattering, and textural modulation by whitecap and breaking waves is not incorporated.

\section{B. Target-to-clutter Contrast Enhancement}

Fig. 4 displays the contrast enhancement between the ship and the surrounding sea as a function of $\beta$ for the three acquisitions in Table I. For each polarization channel, the largest peak indicates maximum contrast enhancement and accordingly the optimal azimuth subaperture bandwidth. The results show that both the optimal subaperture bandwidth and the maximum contrast enhancement varies between the polarimetric channels. The different maximum TCR could be due to the fact that for a given wind speed and incidence angle, the backscatter values over ocean are expected to be higher for $\mathrm{VV}$ than $\mathrm{HH}$, while the $\mathrm{VH}$ and $\mathrm{HV}$ signal is often hidden below the noise-floor [11]. VV polarization has the lowest TCR in all three cases, which is as expected [12].

We also observe variations between the dates in Fig. 4 . There are slight variations in meterological conditions and significant differences in range location, as seen in Table I. The TCR in Fig. 4c is low compared to the two other cases with respect to $\mathrm{VV}$ and $\mathrm{HH}$. A possible reason is the rather low incidence angle $\left(27.1^{\circ}\right)$, which could cause increased backscatter from the background and hence a lower TCR. We expect the target to have different orientations relative to the SAR viewing angle in the various acquisitions. Target signal stationarity, which could be assessed by evaluating the fluctuations of the signal at different azimuth angles of observation [13], needs to be considered. This could have an impact on the location of the peak point and possibly explain some of the fluctations we see in Fig. 4. The contrast is enhanced in all three cases. In Fig. 4, contrast enhancements are achieved for large intervals of $\beta$, often stretching from the no overlap range into the partial overlap range. Contrast enhancements under $\beta>0.5$ would better preserve the spatial resolution.

Judging by the the results in Fig. 4, it seems that a small degree of bandwidth overlap (e.g., $\beta \approx 0.6$ ) may be just as sensible as using no overlap, or a small guarding interval between the subapertures, which has been the practice in some experiments [4]. This goes especially for the HV channel, which is the preferred channel for single polarisation ship detection. However, more empirical studies covering different sea states, incidence angles and ship sizes should be conducted before a firm recommendation can be made. 


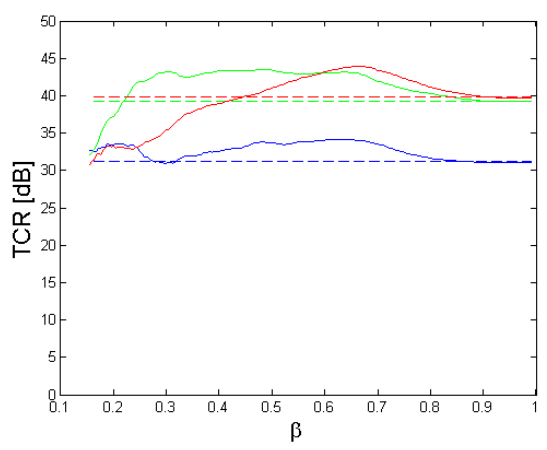

(a) 30 April 2010 .

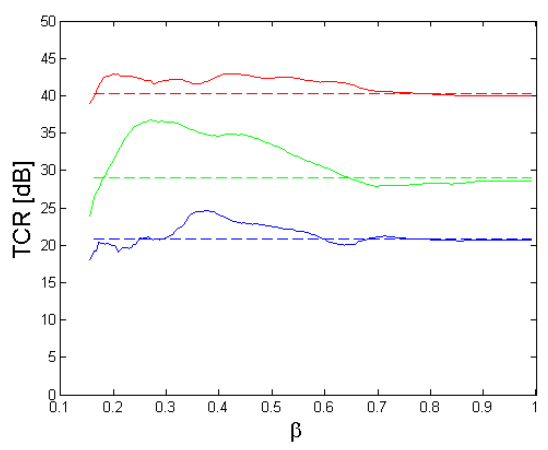

(b) 4 August 2010 .

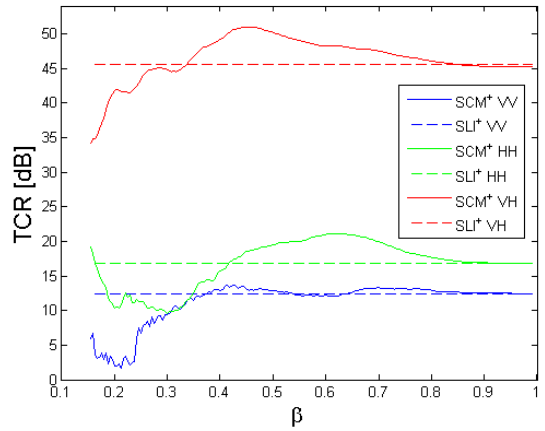

(c) 5 August 2010 .

Fig. 4. TCR versus normalized subaperture bandwidth, $\beta . \mathrm{SCM}^{+}$compared to $\mathrm{SLI}^{+}$for the same ship imaged on three dates in three polarisations.

\section{Spatial Resolution}

Examples of $\mathrm{SCM}^{+}$images and variations in spatial resolution for different values of $\beta$ are shown in Fig. 5. The target acquisition investigated is from the $\mathrm{HH}$ polarization channel of the 5 August 2010 scene. For $\beta=0.3$ severe smearing of the target is evident, but as expected, the spatial resolution is improved for larger $\beta$ values.

At $\beta=0.6$ we are close to the TCR peak point of the $\mathrm{HH}$ curve in Fig. 4c. The corresponding visualization of $\mathrm{SCM}^{+}$is found in the lower left corner of Fig. 5, where we can observe a strong contrast between the target and the surrounding sea. Note that this case, where the subapertures are partially overlapping, provides the best contrast.

The spatial resolution has increased at $\beta=1$, however, the image has a faded appearance due to a smaller dynamic range and lower contrast.
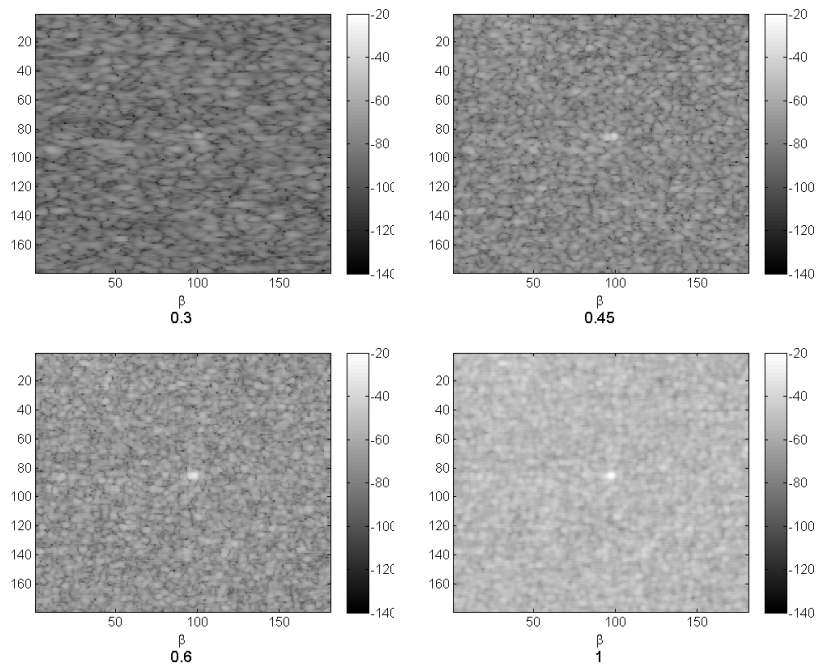

Fig. 5. $\mathrm{SCM}^{+}$products for various values of $\beta$. All images use a common colour scale for direct comparison. The legends are in $\mathrm{dB}$.

\section{CONCLUSIONS AND Future WORK}

An improved version of the subaperture cross-correlation magnitude algorithm for ship detection is presented, which allows overlapping subapertures while mitigating aliasing. The algorithm can also generate single-look intensity products, improved in terms of speckle content. A continuum of subband extraction strategies are investigated, with respect to target-toclutter contrast. Previous studies suggest to apply subapertures that cover half the spectrum each [2]. However, our results reveal contrast enhancements for large ranges of subaperture bandwidths, including partial subaperture overlap. We also observe that the optimal subband extraction strategy is dependent on polarization. Sensor viewing geometry seems to have an impact on the result. Cases with highly agitated open sea states should be investigated in the future.

\section{REFERENCES}

[1] Arnaud, A., "Ship detection by SAR interferometry", Proc. IEEE Int. Geosci. Remote Sens. Symp., Hamburg, Germany, 28 Jun.-2 Jul. 1999, vol. 5, pp. 2616-2618.

[2] Souyris, J.-C., Henry, C., and Adragna, F. "On the use of complex SAR image spectral analysis for target detection: assessment of polarimetry", IEEE Trans. Geosci. Remote Sens., vol. 41, no. 12, pp. 2725-2734, Dec. 2003.

[3] Ouchi, K. and Wang, H., "Interlook cross-correlation function of speckle in SAR images of sea surface processed with partially overlapped subapertures", IEEE Trans. Geosci. Remote Sens., vol. 43, no. 4, pp. 695-701, Apr. 2005.

[4] Johnsen, H. and Larsen, Y., "A novel method for spaceborne SAR vessel detection using complex radar backscatter", ISBN 82-7747-138-6, Tech. Rep., Norut IT, Troms $\varnothing$, Norway, Dec. 2006.

[5] Ouchi, K., Tamaki, S., Yaguchi., H, and Iehara, M. "Ship detection based on coherence images derived from cross correlation of multilook SAR images", IEEE Geosci. Remote Sens. Lett., vol. 1, no. 3, pp. 184-187, Jul. 2004.

[6] Greidanus, H., "Sub-aperture behavior of SAR signatures of ships", Proc. IEEE Int. Geosci. Remote Sens. Symp., Denver, USA, 31 Jul.-4 Aug. 2006, pp. 3579-3582.

[7] Tomiyasu, K., "Phase and Doppler errors in a spaceborne synthetic aperture radar imaging the ocean surface”, IEEE J. Oceanic Eng., vol. 1, no. 2, pp. 68-71, Nov. 1976.

[8] Slade, B., "RADARSAT-2 Product Description", Tech. rep. RN-SP-S21238, iss. 1/6, MDA Ltd., Richmond, Canada, Nov. 2009.

[9] Cumming, I.G. and Wong, F.H., "Digital Processing of Synthetic Aperture Radar Data: Algorithms and Implementation", Artech House, Norwood, USA, 2005.

[10] Oliver, C. and Quegan, S., Understanding Synthetic Aperture Radar Images, 2nd ed., SciTech Publishing, Raleigh, USA, 2004.

[11] Olsen, R.B., Eldhuset, K., Helleren, Ø., and Brekke, C., "Analysis of options for MARISS services extension", GMES Services Element Consolidation for Maritime Security, MARISS-FFITN2-TN-015, Tech. Rep., Nov., 2009.

[12] Touzi, R., Charbonneau, F., Hawkins, R.K., Murnaghan, K., and Kavoun, X., "Ship-Sea contrast Optimization When using polarimetric SARs", in Proc. IEEE Int. Geosci. Remote Sens. Symp., 9-13 Jul. 2001, Sydney, Australia, vol. 1, pp. 426-428.

[13] Ferro-Famil, L. and Pottier, E., "Urban area remote sensing from Lband PolSAR data using Time-Frequency techniques", in Proc. Urban Remote Sens. Joint Event, Apr. 2007, Paris, France, 6 pp. 